\title{
Le débat méthodologique sur l'enseignement des langues étrangères dans les pages du Boletín de l'Institución Libre de Enseñanza (BILE)
}

\section{Carmen Roig Morrás}

\section{(2) OpenEdition}

\section{Journals}

Electronic version

URL: https://journals.openedition.org/dhfles/3047

DOI: $10.4000 /$ dhfles.3047

ISSN: 2221-4038

\section{Publisher}

Société Internationale pour l'Histoire du Français Langue Étrangère ou Seconde

\section{Printed version}

Date of publication: 1 June 1999

Number of pages: p.227-247

ISSN: 0992-7654

\section{Electronic reference}

Carmen Roig Morrás, "Le débat méthodologique sur l'enseignement des langues étrangères dans les pages du Boletín de l'Institución Libre de Enseñanza (BILE)", Documents pour l'histoire du français langue étrangère ou seconde [Online], 23 | 1999, Online since 24 May 2015, connection on 10 March 2023. URL: http://journals.openedition.org/dhfles/3047 ; DOI: https://doi.org/10.4000/dhfles.3047

This text was automatically generated on 10 March 2023.

All rights reserved 


\title{
Le débat méthodologique sur l'enseignement des langues étrangères dans les pages du Boletín de l'Institución Libre de Enseñanza (BILE)
}

\author{
Carmen Roig Morrás
}

1 Dansla seconde moitié du XIX ${ }^{e}$ siècle, partout en Europe l'enseignement deslangues étrangères vivantes (désormais LEV) connaît un essor considérable lié à l'apparition de nouveaux besoins socio-économiques et au désirgénéralisé de moderniser le pays. D'abord en Allemagne, en France à sa suite, l'objectif pratique de cet enseignement devient prioritaire : les LEVsont conçues comme des instruments de communication dans un espacesans frontières, et il ne s'agit plus d'apprendre la langue littéraire mais d'apprendre àparler dans un idiome étranger. Dans le système scolaire, la nécessité d'introduire la pratique orale en classe de LEV bouleverseles pratiques d'enseignement et la conception des manuels. Dans ce contexte naît ce qu'on peut qualifier de révolution méthodologique, qui débouche àla fin du siècle sur la méthode directe, selon la dénomination laplus répandue, bien qu'elle ne soit pas la seule. Au-delà des frontières, en Allemagne, en Angleterre, en France, la réforme de l'enseignement des LEVcrée un espace commun où les échanges se multiplient entre linguistes, phonéticiens, pédagogues et méthodologues. Ce mouvement d'idées n'estpas étranger aux universités qui jouent un rôle de premier ordre dans la définitiondes nouveaux objectifs, contenus et principes théoriques de cet enseignement. Les résistances des enseignants, les débats méthodologiques, les polémiques et critiques de toute sorte marquent cette période d'effervescence.

2 Ce bouillonnement, hélas! ne franchit pas les Pyrénées. Il est vrai qu'en Espagne, comme ailleurs, l'enseignement des LEV s'intensifie pendant la même période. Dès 1857, la Ley Moyano sanctionne son intégration dans le système scolaire, en théorie du 
moins, car dans la pratique cette intégration ne sera effective que vers la fin du siècle. En même temps les Ecoles de Commerce jouent un rôle pionnier dans la promotion semi-officielle des cours de LEV à objectif pratique. Parallèlement on assiste à une floraison d'académies, écoles et cours privés qui essayent de répondre à une demande sociale sans doute importante. Pendant ce temps, les langues étrangères restent toujours absentes de l'université, qui leur refuse obstinément le statut de discipline académique ; où trouver alors les spécialistes motivés et qualifiés pour participer, ou tout au moins pour s'intéresser - à ce vaste mouvement européen ? Aussi, en Espagne, l'enseignement des LEV poursuit-il sa route jusque bien avant dans le $\mathrm{XX}^{\mathrm{e}}$ siècle comme un navire en dérive. L'évolution méthodologique quasi-inexistante est livrée à l'empirisme des praticiens plus ou moins habiles, et reste tributaire d'une tradition surannée, ou des apports étrangers, sans que dans le cadre du système scolaire on puisse déceler des initiatives de portée générale, encore moins une réflexion théorique autonome.

3 C'est dans ce contexte de vide méthodologique que l'action de l'Institution Libre de Enseñanza (ILE) constitue l'exception. Nous avons eu précédemment l'occasion de réfléchir dans les pages de Documents (Roig : 1990, 1998) sur le rôle décisif joué par l'ILE dans l'intégration définitive des LEV, et notamment du français, dans le système scolaire espagnol. Dans ce travail nous nous proposons d'analyser les différents aspects de la réflexion théorique et méthodologique qui a accompagné cette action, et en même temps, d'exhumer quelques textes qui ont présidé à la naissance, en 1931, de la Section de Filología Moderna (tipo B a base de Francés) comme discipline académique de l'université espagnole. Car la réflexion méthodologique est indissociable de l'intégration des langues étrangèresvivantes au sein de l'université, ainsi que de la formation universitaire spécifique des futurs professeurs de français du secondaire.

Dès sa création en 1876, la promotion de l'étude des LEV est restée une préoccupation constante desresponsables de l'ILE qui dans leur action ont essayé de définirobjectifs» moyens et méthodes. Nous avons consulté le Boletín de l'Institución(BILE) dans l'espoir d'y trouver les traces de cette réflexion. En effet, créé en 1877, le BILE est la tribune de l'actualité en matière éducative en Europe et, jusqu'en 1936, il s'est fait l'écho de tout ce qui est lié au mouvement de rénovation pédagogique, notamment en Franceet en Belgique. Le résultat decette consultation estdécevant, tellement laréflexion sur l'enseignement des LEV est insignifiante dans ses pages, à des dates où ledébat est à sonapogée. L'effervescence qui acaractérisé la seconde moitié du siècle est passée soussilence dans lapremière revue pédagogique du pays, et il faudra attendre les années 20 pour y retrouver les traces d'undébat initié ailleurs cinquante ans plus tôt. Avantde lesanalyser, rappelons pour mémoire les rares contributions qui les ont précédées.En 1882, un compte rendu anonyme présente l'opusculeintitulé El Método en la enseñanza de las lenguas qui, après avoir disqualifié la prétention des méthodespratiques d'assimiler l'apprentissage dela langue étrangère à celui de lalangue maternelle, propose commenouveauté méthodologique la philologie et lecomparatisme ( BILE : 278). Danslamême année, le rapport surl'organisation desétudes commerciales» élaboré par la Junta Facultativa de laInstitución à la demande de l'Asociación de Profesores Mercantiles insiste sur lanécessité de l'étude deslangues vivantes à des finspratiques(BILE : 83). En 1902, un compte rendutraduit de l'étranger, d'unarticle du professeur Jespersen, de l'université de Copenhague, témoigne d'un début d'ouverture vers ce débat méthodologique qui nous occupe. Il y est question de " método naturel », de « fonética práctica», de « alfabeto fonético » (BILE : 111-112). En 
1912 les «Observaciones sobre laenseñanza del Inglés » (BILE : 193-199), de A. Pestafia, constituent la première contribution nationale à ce débat. Sonauteur, professeur d'anglais» après un séjourd'études en Angleterre» présente lesystème éducatifanglais» et notamment l'enseignement de lalangue et de lalittérature auxjeunes écoliers. Sous saplume, l'anglais prend la place du latin comme langue de culture, de formation, de discipline intellectuelle. Ensuite, elle extrapole et propose ce modèle à tous les pays, non pas pour apprendre la langue maternelle, mais pour apprendre l'anglais comme langue étrangère. D'après l'auteur

...el aprendizaje del inglés, tal como hoy se practica en Inglaterra, y como en realidad debe practicarse en todas partes, tiene incontestablemente un valor excepcional como agente formativo del gusto y de todas las virtudes (196).

Les latinistes de la Ratio Studiorum n'auraient pas fait mieux. En somme, l'article est un bon indice du niveau de qualification de nos enseignants de LEV à l'époque.

En 1921, la réflexion d'Américo Castro, alors Catedrático à l'Universidad Central de Madrid, ouvre la voie de la modernité et de la rationalité dans le débat sur le statut des langues vivantes dans notre pays. Sous le titre « La enseñanza de las lenguas modernas » (BILE : 119-121), il rappelle l'urgence de la création à la faculté des Lettres de Madrid d'une section destinée au moins à l'enseignement du français. Sa préoccupation est le fruit de sa propre expérience. Celle de professeur universitaire de prestige international qui, lors de ses contacts avec ses pairs étrangers, s'est vu contraint d'avouer à des collègues stupéfaits que l'université espagnole ignore totalement « el cultivo de las lenguas y literaturas modernas ».

7 La France apparaît à ses yeux comme le modèle à suivre. Chez nos voisins, il observe que presque toutes les universités possèdent des chaires d'anglais occupées par des spécialistes de prestige qui, à la parfaite maîtrise de la langue anglaise, ajoutent la connaissance de la littérature et de l'histoire du pays. Autour d'eux, les jeunes générations se forment à la recherche et produisent toute sorte de publications. De plus, cette étude scientifique, et somme toute désintéressée et quelque peu élitiste des langues modernes, n'est pas la seule à occuper les activités de ces sections. A. Castro rappelle également le côté pratique de cet enseignement : l'université est la pépinière des futurs enseignants des langues vivantes du secondaire. Ce qui l'amène à comparer la formation de ces professeurs, formes à l'université, capables de parler et d'écrire correctement la langue et possédant une bonne connaissance de la civilisation française, à celle de leurs homologues espagnols, troupe bariolée de chanoines, marchands, ou maîtres de langues, qui souvent n'ont jamais mis le pied à l'université. Dans le meilleur des cas, il s'agit d'universitaires espagnols, formés dans d'autres disciplines, Catedráticos de leur spécialité et chargés en même temps des rares cours de français où ils enseignent une langue qu'ils ne connaissent qu'en amateurs.

Aussi, rappelle notre professeur, les jeunes bacheliers français sont-ils mieux préparés que les espagnols pour accéder à l'université, ou tout simplement pour s'engager dans la vie professionnelle. Dans le système espagnol, au contraire, l'étude des langues étrangères n'a pas dépassé le stade de simple ornement. Et A. Castro reproche vertement au ministère de se limiter « a mantener una apariencia de enseñanza en los Institutos y Escuelas de Comercio » consistant en deux ans de français et une année d'anglais ou d'allemand, alors qu'il est du domaine public que personne n'a jamais appris, même pas à lire, une langue étrangère dans ces établissements, c'est-à-dire «que ni aun la finalidad mas modesta y elemental se logra dentro de nuestra enseñanza » et 
que les élèves espagnols « abandonados a sí mismos los [los idiomas] aprenden aisladamente cuando la necesidad les apremia ».

Dans sa brièveté, l'intervention de Castro a le mérite d'être le premier diagnostic lucide du mal, qu'il dénonce dans ses trois aspects essentiels étroitement liés : l'inexistence de professeurs qualifiés pour enseigner les langues étrangères, le statut dévalorisé de ces enseignements, qui en est la conséquence, et l'absence de sections universitaires consacrées à l'étude des langues vivantes, qui est à l'origine du problème. C'est le parfait cercle vicieux qu'il entend rompre une fois pour toutes, car A. Castro sachant que la conjoncture politique est favorable aux réformes éducatives, exige des pouvoirs publics des mesures urgentes pour changer cet état des choses:

España tiene cada dia mas relaciones internationales, cada vez crece el numéro de Cátedras de español en los Estados Unidos, Inglaterra, Italia, Francia etc. Y, especialemente para la Facultad de Letras, es motivo de sonrojo ver que no sale de ella una pagina sobre una civilización extranjera, que no se dice allá una palabra sobre Dante, Shakespeare o Molière, que sus estudiantes retroceden espantados ante un libro en inglés o alemán, y que solo alguno que otro maltraduce el francés didáctico [...]?,No sería hora de que la Facultad de Letras de Madrid poseyera, por lo menos, una decorosa enseñanza del francés ? ${ }^{1}$ (121).

10 Finalement c'est l'argument de prestige qui vient couronner son plaidoyer pour les langues vivantes. L'expansion de l'espagnol exige la connaissance des langues et littératures des pays où l'intérêt pour notre langue se consolide. Réciprocité oblige sans doute. Paradoxalement la diffusion de notre propre langue devient prétexte à celle du français.

11 Le principal intérêt des observations de Castro est d'avoir insisté sur l'origine du mal, qui se trouve à l'université, et non pas dans l'enseignement secondaire, qui n'en est que la conséquence. C'est parce qu'au sein des facultés des Lettres on ignore « el aspecto más elevado, científico, en que el fin que se persigue es el estudio de la lengua en sí misma (gramática), o de la cultura expresada en ella (historia literaria, etc.) », que le statut des enseignants et de la discipline dans le système scolaire est au plus bas de l'échelle et que le niveau de connaissance des langues vivantes des étudiants espagnols, et même des professeurs universitaires, est déplorable. Aussi, à l'instar de tous les pays modernes qui lui servent de modèle, propose-t-il d'inverser les priorités et de commencer par la création de sections de langues étrangères dans les facultés des Lettres. Ce n'est qu'à cette condition que l'on peut sérieusement envisager la modernisation de l'enseignement de ces langues dans le secondaire. Cet appel urgent adresse à des responsables politiques, favorables à l'époque aux idées novatrices en matière d'éducation, ne tombe pas dans le vide. Mais, dans l'immédiat, les priorités du ministère s'orientent vers les niveaux primaire et secondaire et la future section de Philologie française de l'Université de Madrid devra attendre jusqu'à 1931.

En attendant, les pages du BILE deviennent la tribune où, très timidement encore, s'initie le débat méthodologique sur les langues vivantes. En 1925 et 1926 le Boletín publie deux articles d'intérêt pour notre propos. Le premier, sous le titre " La enseñanza de las lenguas extranjeras " est la traduction in extenso de l'article de Jespersen objet du compte rendu commenté plus haut. Présenté comme une nouveauté, il s'agit en fait d'un travail paru à la fin du XIX ${ }^{e}$ siècle qui se fait l'écho des débats suscités par la réforme des enseignements des langues vivantes en France et en Allemagne. Au-delà des polémiques déjà dépassées, deux aspects abordés par le professeur Jespersen sont encore actuels. D'abord la défense du nouveau statut des 
langues vivantes dans les systèmes éducatifs et l'affirmation de leur autonomie face aux langues classiques, ainsi que de leur spécificité comme discipline scientifique. Ensuite une redéfinition des objectifs pratique et culturel au sens très large. L'exposition détaillée de la méthode directe est d'un intérêt moindre à cette date. Il expose ses principes théoriques, ses objectifs, sa méthodologie. Pourtant, en 1925, cette apologie est déplacée, puisqu'on connaît déjà les effets pervers qui ont résulté de son application stricte. Ce qui ne signifie pas qu'une telle information soit superflue pour le lecteur espagnol. Resté à l'écart du débat méthodologique autour des langues vivantes, il méconnait les données essentielles du problème. Il peut lui être utile de connaître les aspects autant positifs que négatifs des expériences de nos voisins qui désormais vont constituer notre modèle.

13 A peine quelques mois plus tard, et sous un titre presque identique, « La enseñanza de las lenguas vivas ", le professeur Rubén Landa publie dans la même section un long article qui marque le contrepoint du précédent (BILE, 1926). Son auteur, Catedrático de Psychologie au lycée de Sala-banque, appartient à cette élite qui, sous les auspices de la Junta para ampliación de Estudios, a bénéficié d'un long séjour d'études en France et en Angleterre, où il a été chargé de s'informer sur les systèmes éducatifs de ces pays et notamment sur l'enseignement des langues vivantes. Il y a suivi des cours destinés à la formation méthodologique des professeurs de langues étrangères dans les universités de Londres et Paris et, en qualité d'observateur, il a assisté à des cours de langue vivante dans les établissements scolaires de ces pays. Il peut également se vanter d'avoir appris plusieurs langues, et aussi de les avoir enseignées, ce qui lui permet de juger de l'efficacité des différentes méthodologies et de les évaluer en connaissance de cause.

14 Sans la prétention d'innover, dès les premières lignes le but de son intervention est clairement défini :

No pretendo dar a conocer nada nuevo sino únicamente como entiendo que deberia aplicarse en nuestros Institutos de Segunda enseñanza y en nuestras Escuelas Normales algo ya muy conocido en el mundo, aunque apenas practicado en Espana : los métodos relativamente modernos de la enseñanza elemental de las lenguas vivas. $^{2}(35)$.

De ce projet d'adaptation au système éducatif espagnol des méthodologies modernes pratiquées en Europe, nous retenons plusieurs aspects qui configurent ce qui désormais va constituer la singularité de l'option méthodologique nationale. D'abord nous assistons à une redéfinition des objectifs de cet apprentissage. L'auteur, après avoir reconnu que l'apprentissage d'une langue vivante exige la maîtrise des quatre compétences : parler, écrire, comprendre la langue orale et la langue écrite, revendiquées par tous les tenants du renouveau, il élimine les trois premières pour conclure que « el objetivo de esta enseñanza en nuestros Institutos y Normales debe ser principalmente capacitar para leer, para entender la lengua escrita » (36). Sous le signe du renouveau et de la modernité, nous assistons à la récupération du vieux discours pragmatique des "Ilustrados" du XVIII siècle (Roig, 1994 : 38-39). Les langues étrangères vivantes restent toujours piégées dans leur rôle ancillaire d'instrument d'accès à la connaissance, seul objectif noble. Une fois de plus, les langues modernes se substituent au latin. On les apprend:

Para adquirir un instrumento de cultura. Tanto es su valor en este sentido que puede decirse que un español que no sabe francés, ni inglés, ni alemán no es un hombre culto. Asi sucedia antiguament eon el latin. ${ }^{3}$ (36). 
Dans la pratique, ce plurilinguisme proclamé sur le plan des principes, se réduit à la connaissance du français, puisque cette langue « nos permite participar de la cultura universal, traspasar las puertas de nuestra casa y vivir en el mundo »(36). Il y a pourtant du progrès, car cette connaissance ne devrait pas se limiter à la langue, à l'instrument, mais, dans la mesure du possible, elle devrait s'étendre à la culture française au sens large. A cet effacement des trois objectifs spécifiques prônés par les tenants de la réforme, correspond l'incorporation d'un nouvel objectif, tiré des arguments des défenseurs de l'étude des langues classiques et vieux de plusieurs siècles. Ainsi notre auteur souligne que grâce aux exercices de traduction « la clase de lenguas vivas puede servir para adquirir un dominio mayor de la lengua materna y como gimnasia para desarrollar la inteligencia »(36). Dans les pages suivantes le professeur Landa s'étend longuement sur les qualités formatives de l'étude des langues vivantes au même titre que celle du latin et du grec, et regrette que cet aspect ait été négligé par les théoriciens de l'enseignement moderne. Sur ce point, sa critique se centre sur la réforme française de 1902 qui, à ses yeux, échoue dans sa prétention de placer le baccalauréat moderne sur le même pied quele classique parce que :

Al dar a la enseñanza de las lenguas vivas un carácter exclusivamente práctico y al imponer en ella únicamente el método directo, la coloca en una situation de inferioridad con relation a la enseñanza clásica, pues limitando su objetivo a la adquisición de un mero instrumento, no es posible obtener con ella, como con la enseñanza clásica, resultados considérables en cuanto a la lengua materna y a la formation de la inteligencia. ${ }^{4}$ (37).

Il est surprenant, qu'en 1926, R. Landa se limite à critiquer la méthode directe, déjà battue en brèche en France comme ailleurs, et passe sous silence le mouvement d'assimilation des langues modernes aux langues anciennes qui s'impose en France depuis dix ans et constitue, avec la revendication des Humanités classiques, la nouveauté méthodologique la plus proche de ses vues.

Cet infléchissement des objectifs, signalé plus haut, s'accentue lorsque notre auteur en arrive à comparer leurs méthodologies respectives. Pour lui la méthode directe " no tiene valor alguno para la formación de la inteligencia » puisqu'elle exclut la réflexion, c'est-à-dire, toute activité intellectuelle. Son seul mérite est d'avoir découvert que l'apprentissage d'une langue vivante s'appuie sur l'imitation et la répétition. On apprend la langue comme on apprend « a escribir a máquina o a montar en bicicleta ». La traduction au contraire exige un grand effort intellectuel et l'enseignement classique tire tout son prestige de ces exercices rigoureux. C'est pourquoi l'enseignement moderne ne parviendra à l'égaler qu'en l'imitant sur cet aspect (37).

19 A partir de ces présupposés théoriques R. Landa en vient à exposer ce qui est le but déclaré de son intervention, c'est-à-dire, leur application pratique à renseignement des langues vivantes dans les établissements scolaires espagnols. La méthodologie proposée est double et correspond aux deux objectifs retenus. La méthode directe convient à l'acquisition de la langue, de l'instrument, mais l'objectif de formation intellectuelle exige la pratique intensive de la traduction. La première se limite aux deux premières années d'apprentissage. Il en fait une description rigoureuse et détaillée qui n'omet aucun des avantages attribués à la méthode et témoigne d'une bonne connaissance du débat instauré autour d'elle. Mais, au-delà de son apparente objectivité on sent percer un malaise profond, le rejet, non pas d'une méthode, dont les résultats seraient plus ou moins satisfaisants, mais d'une conception de l'éducation qui lui semble incompatible avec son credo philosophique. Contre ceux qui préconisent la répétition mécanique, la 
mémorisation, il oppose une éducation centrée sur la réflexion, la maîtrise des concepts et des principes théoriques :

La diferencia estriba, en último caso, en el valor que se da a la réflexion para aprender algo. Por una parte se dice : a hacer algo se aprende reflexionando antes acerca de nuestra acción, pensando antes cómo se hace, conociendo los conceptos y leyes générales referentes a esa actividad, es decir su teoria. Por otra parte se dice : a hacer algo se aprende viéndolo hacer y haciéndolo ; no es el conocimiento de la teoria, sino la práctica ; no es la reflexión acerca de la acciôn, sinola action misma, lo que nos ensena a hacer [...] La répétition y el hábito, no el conocimiento ni la reflexión es lo que enseña a hacer, lo mismo a hablar una lengua que a tocar el piano o a nadar. ${ }^{5}$ (38).

20 En dernier ressort, la différence réside dans la valeur accordée à la réflexion pour apprendre quelque chose. Certains disent : on apprend à faire quelque chose en réfléchissant d'abord au sujet de notre action, il faut penser à la façon de faire, connaître les concepts, et les lois générales relatives à l'activité en question, c'est-àdire, sa théorie. D'autre part on dit : on apprend à faire quelque chose en observant ceux qui le font et en le faisant; ce n'est pas la connaissance de la théorie, mais la pratique ; ce n'est pas la réflexion au sujet de l'action, mais l'action en elle-même qui nous apprend à faire [...]. La répétition et l'habitude, et non la connaissance ni la réflexion nous apprend à faire, il en est de même pour parler une langue que pour jouer du Piano ou nager.

21 R. Landa voit la nécessité d'adapter une méthode qui - avec toutes ses limites - a déjà fait ses preuves et se montre le seul instrument susceptible d'introduire un changement dans la pratique de nos classes de langue. Mais il éprouve une forte réticence à se ranger par ce biais sur une conception de l'apprentissage ouverte à tous les excès et qui conduirait au bannissement de la réflexion personnelle. Dans ce tiraillement, mieux vaut pour ce psychologue férude classicisme, couper la poire en deux, et adopter la méthode directe, mais en la rattachant, non pas à cette modernité de mauvais aloi, mais à la vieille tradition classique chargée d'humanisme.

Aussi, en conclusion deson analyse de la méthode, propose-t-il " la description mas perfecta que conocemos del método directo ", tirée des Confessionsde Saint-Augustin (Livre I, chap. VIII : « Del modo como aprendió a hablarcuando llegó a la niñez ») :

Cum ipsi [les autres] appellabant rem aliquam, et cum secundum eam vocem corpus ad aliquid movebant, videbam et tenebam hoc ab eis vocari rem illam, quod sonabant : cum eam vellem ostendere [...] Ita, verba in variis sententiis, locis suis posita, et crebo audita, quarum rerum signa essent, paulatim colligebam, meas que jam voluntatis edoni to in eis signis ore, per haec enuntiabam. ${ }^{6}(39)$.

La citation, est en effet,une excellente illustration de l'apprentissage enfantin de la langue maternellequi contient « toda la esencia del método directo » (39) et à laquelle ilne manque que l'application à l'apprentissage de la langue étrangère. Parée de ces nouvelles lettres de noblesse, la méthode directe peut désormaiss'inscrire dans renseignement moderne sans porter atteinte auxprincipes fondamentaux de l'éducation classique, ce qui accroît d'autantson prestige. Il faut admettre l'originalité d'une telle filiation, etce n'est pas laseule, car, toujours fidèle à ses préjugés, ilenvisage la possibilité de voir un jour les enseignements des langues vivantes et des langues mortes suivre des démarches parallèles et adopterla même méthodologie. Les deux adopteraient la méthode directepour l'apprentissageinstrumental des deux premières années, réservant la pratiquede la traduction pour les cours avancés : " y entonceses cuando, colocadasen igualdad de condiciones, podrà juzgarse desu valor relativo » (37). 
24 Mais, en fait, qu'est-ce quenotre expertentend par cet apprentissageinstrumental siréitéré ? Il n'envisage pas la maîtrise de lalangue» ou 1'acquisitiond'un vocabulaire ample, maisce qu'ilpropose comme objectif n'est pas moins ambitieux :

Lo esencial es que el alumno sehaya asimilado la estructurade la lengua, que piense en la lengua extranjera, que pueda, sin cuidarsede ello, como por un instintogramátical, construir lasfrases en el orden debido y usar las palabras variables con lasmodificaciones que corresponde en cada caso. ${ }^{7}(41)$.

Rien, dans cetteformulation, ne nous permet de savoir s'il parle d'une compétence exclusivement écrite ou sil'expression orale est également envisagée. Non plus àquel niveau situele professeur Landa celte expression spontanée, fruit d'un instinct grammatical et d'une maîtrise morphosyntaxique acquises en deux ans. Onreste rêveur devant le caractère ambitieux et volontariste de tels objectifs. Ilest également difficiled'établir le lien direct entre cetteassimilation visant l'expression - la construction de phrases -, et la compétence de lecture proposée au départ comme seul objectif linguistique de cet apprentissage. Le caractère contradictoire de la démanche de notre expert s'accentue de plus en plus. Son soucide modernité voit dans la méthode directe une voie incontournable vers la maîtrise de la langue, et en même temps une menace pour le prestige de cet enseignement qu'il a l'ambition d'instaurer définitivement. Aussi s'efforce-t-il de l'apprivoiser en lui cherchant des filiations classiques» en lui assignant des compagnons de route distingués, en la réduisant à sa part congrue au profit d'objectifs plus élevés.

En effet, une fois atteint l'objectif instrumental en deux ans, c'est sur le renouvellement de la pratique de la traduction que s'appuie son projet de modernisation. Grâce aux exercices de traduction, renseignement des langues vivantes sera en mesure de rivaliser avec celui des langues classiques pour atteindre les trois objectifs traditionnels qui lui donneront tout son prestige : la connaissance de la culture étrangère, la maitrise de la langue maternelle et la formation morale et intellectuelle. C'est pourquoi, c'est à la traduction que doit revenir la part du lion dans la programmation horaire :

Mientras menos se tarde en adquirir el instrumento y más tiempo pueda dedicarse a la traducción, mejor. El método directo que pide un trabajo meramente mecánico, sin valor formativo alguno, conviene pues emplearlo únicamente durante el tiempo estrictamente . indispensable. ${ }^{8}(41)$.

27 En somme, le projet d'adaptation de R. Landa relève, même s'il ne le dit pas, d'un éclectisme initié en France vingt ans plus tôt, issu de la nécessité d'adapter la méthode directe aux objectifs culturels et formatifs exigés par le second cycle. Comme chez nos voisins, la méthode proposée par notre auteur veut être un compromis entre la méthode directe et la méthode traditionnelle. Pourtant, dans le cas qui nous occupe, on a l'impression que l'équilibre qui permettrait de corriger les carences et les excès de l'une et de l'autre n'est pas atteint. A l'horizon de sa réflexion, l'ombre des langues classiques se profile comme le seul référent de prestige et sa construction méthodologique s'enlise dans la confusion de concepts, objectifs et pratiques.

Le projet de formation des futurs professeurs de LEV abordé dans la deuxième partie de cet article est l'aboutissement naturel de cette réflexion. Sa perspective est résolument pratique et rejoint dans ses conclusions celle d'Américo Castro commentée plus haut. A la condamnation de la situation présente, suit la réclamation pour la faculté de Lettres dé Madrid d'une section consacrée àl'étude des langues et littératures modernes et chargée de la formation des enseignants. En attendant, il propose une formation universitaire partagée entre nos facultés et l'Institut français de Madrid. Cette 
formation comprendrait, à peu près à égalité, les langues et littératures espagnole et française. Ces dernières seraient dispensées par un professeur espagnol versé dans le domaine, secondé par un lecteur français chargé des cours de conversation. Le cursus serait complété par un cours dephonétique espagnole, dispensé au Centro de Estudios Históricos, en guise de préparation à l'étude ultérieure de la phonétique française qui serait menéeen .France. Au total, une formation universitaire en Espagne de quatre années, complétée par un séjour de deux ans à l'étranger. L'auteur suggère des contenus novateurs pour ce séjour. Une année à Paris pour suivre les cours de la Sorbonne destinés à la formation des professeurs de français à l'étranger, sous la direction de F. Brunot. Cette formation théorique serait complétée par une formation méthodologique consistant en des stages pédagogiques pratiques auprès de professeurs en exercice.

Pour la deuxième année, ou du moins pour une partie, il conseille l'approfondissement des aspects méthodologiques. Aussi, propose-t-il de quitter la France afin de visiter les pays et les établissements scolaires dans lesquels le français est enseigné comme langue étrangère. Il observe d'ailleurs queles cours de la Sorbonne, excellents pour l'étude de la langue et de la littérature, négligent les aspects méthodologiques, si essentiels dans l'enseignement des premières années. Son choix se porte sur les cours d'été de l'University Collège destinés aux professeurs de français sous la direction du professeur Jones, ${ }^{9}$ première autorité en matière de phonétique.

30 Il reste à se demander quelle a été l'incidence réelle de cet effort de rénovation méthodologique, discutable peut-être, mais lucide et sérieux, et qui a le mérite d'être le premier depuis l'intégration des langues vivantes dans le système scolaire. Les limites de ce travail nous empêchent de suivre ses traces dans Je détail. Rappelons brièvement l'écho des idées de R. Landa à trois moments différents. D'un côté l'article est une systématisation de la méthodologie appliquée dans l'enseignement du français au sein de l'Instituto-Escuela, telle qu'elle figure dans le bilan élaboré en 1925, à la fin de cet essai pédagogique initié en 1918 (Roig :1998). Nous y retrouvons une totale identité de vues quant à filiation méthodologique, objectifs et pratiques de classe. Peu importe que R. Landa ait été ou non l'inspirateur direct de cet essai pédagogique. Toujours est-il que l'expérience menée à l'Instituto-Escuela ayant été concluante, son article de 1926 vient lui apporter sa caution scientifique, en en faisant une exposition rigoureuse et détaillée adressée à un public plus large. Finalement, avec des modifications orientées vers le rééquilibrage des quatre objectifs, ces propositions méthodologiques passeront dans les instructions officielles concernant les langues vivantes lors du Plan d'études approuvé par le gouvernement républicain en 1934. Et il n'est pas superflu de rappeler que ces instructions sont les seules qui, jusqu'à l'arrivée des méthodes audiovisuelles, aient donnée une cohérence méthodologique à ces enseignements. Vient ensuite l'Ordre ministériel de 1927 publiant les Cuestionarios des langues vivantes. Ce texte établit le programme officiel destiné à déterminer les contenus des manuels des quatre langues figurant dans le cursus scolaire : français, anglais, allemand et italien, bien que seul le questionnaire de français soit réellement développé. Le questionnaire est précédé d'une exposition théorique et d'orientations méthodologiques communes aux quatre langues, et suivi de directives précises sur les pratiques de classe conseillées, dans lesquelles le recours à la méthode orale est constamment invoqué. Sans le nommer, ces directives réintroduisent en force l'objectif pratique. L'éclectisme domine l'ensemble de ces orientations, qui dénotent une volonté de renouveau et d'équilibre entre les excès de signe opposé. Quant au questionnaire proprement dit, composé de 59 thèmes 
de vocabulaire et autant de grammaire, il marque le retour aux anciens schémas et semble contredire le corps de doctrine qui l'accompagne.

31 En somme, il s'agit d'un texte de compromis dans lequel deux discours parallèles se superposent sans arriver à s'interpénétrer. D'un côté la volonté ministérielle de renouveler l'enseignement des LEV, exprimée à travers la réflexion méthodologique initiale et les orientations pratiques de la fin, élaborées par la commission, s'inspirent des même principes que le projet de R. Landa. De l'autre, les contenus de grammaire et de vocabulaire proposés pour les manuels introduisent une dissociation entre les buts et les moyens et dénoncent la persistance des vieux schémas de l'enseignement traditionnel. Ces questionnaires, proposés par les Catedráticos de français, - la commission s'étant limitée à les examiner et à donner son approbation - traduisent ce qu'était à l'époque l'enseignement du français dans les centres officiels et l'incapacité des enseignants de se moderniser.

Finalement, le Décreto du 15 septembre 1931 établit à titre d'essai dans les facultés de Lettres de Madrid et Barcelone des épreuves conduisant à une licence en Philologie moderne, option espagnol ou toute autre langue étrangère, qui paraissent conçues pour sanctionner la formation acquise par les candidats qui auraient suivi le programme élaboré par R. Landa cinq ans plus tôt. Les candidats, après avoir suivi trois années d'études en faculté de lettres doivent passer deux séries d'épreuves. La première, commune à toutes les philologies modernes, comprend pour moitié les langues classiques et l'arabe et des thèmes de philosophie, histoire et littérature espagnoles. La deuxième série est spécifique aux langues étrangères. Celle de français comprend des exercices écrits et oraux. Parmi les premiers : version latine, version et thème français accompagnés de leur transcription phonétique et deux dissertations, l'une de littérature espagnole, l'autre, en français, de littérature française. Les exercices oraux consistent en des commentaires philologiques d'un texte espagnol et de deux textes français, l'un en français moderne, l'autre en ancien français suivis de deux exposés, l'un sur la littérature espagnole, l'autre sur la française. Tous les exercices oraux concernant soit la langue, soit la littérature françaises se feront dans cette langue. Il reste une dernière épreuve de version d'une deuxième langue vivante au choix du candidat. Si on tient compte de la durée prévue pour chacun des exercices, les épreuves spécifiques en langue française représentent à peu près $50 \%$ du total, ce qui, en théorie, permet de juger de l'aptitude du candidat, même si on doit regretter le poids excessif des langues anciennes et le silence sur la formation méthodologique. Le " Proyecto de Ley de Bases de la reforma universitaria » du 14 mars 1933 propose l'intégration définitive des études de «Filología Moderna » option français, dans les universités de Madrid et Barcelone, et sanctionne sans modification, ce modèle d'épreuves.

Une conclusion semble se dégager de cette analyse du BILE. La rareté des sources, leur manque d'originalité et l'absence de véritable débat à plusieurs voix, ne doivent pas minimiser l'importance de ces contributions. Elle est grande si on la mesure à l'influence qu'elles ont eue sur la modernisation de l'enseignement des LEV, et notamment du français, dans le système scolaire espagnol. En ce sens les articles de A. Castro et de R. Landa représentent deux apports décisifs à la constitution des langues modernes comme discipline académique dans notre pays. Le discours du premier a une visée essentiellement scientifique. C'est le discours d'un universitaire qui envisage l'étude des langues et littératures étrangères comme une fin en soi dont l'objectif est le 
savoir, la recherche scientifique. La visée pragmatique n'est pourtant pas négligée puisqu'à ses yeux la création de sections de langues étrangères est inséparable de la formation des futurs enseignants. Cette visée pragmatique est approfondie par R. Landa, dont l'article marque le premier pas vers la constitution d'une véritable didactique des LEV Certes, on peut lui reprocher son asservissement au modèle des langues classiques. Ses préjugés, qui le conduisent à une dévalorisation de l'enseignement pratique et à une formulation d'objectifs contradictoires et incompatibles avec le but déclaré de modernisation de ces enseignements. Mais il faut également mettre à son acquis d'avoir, le premier, pris conscience de la spécificité et l'importance de la discipline, et des questions de méthode, d'avoir hissé les langues étrangères à la hauteur du latin dans le nouveau baccalauréat. Son projet de formation universitaire des futurs professeurs de français témoigne de sa préoccupation pour assurer leur formation méthodologique, ce qui constitue une grande nouveauté dans le système universitaire espagnol. Sur la longue durée, on peut mieux évaluer l'importance de ces initiatives qui sans la coupure de la guerre civile auraient pu assurer l'entrée définitive des langues vivantes étrangères dans la modernité.

\section{BIBLIOGRAPHY}

\section{Ouvrages consultés}

CACHO VIÛ, V. (1962), La Institución Libre de Enseñanza I. Origenes y etapa universitaria, Madrid, Rialp, pp. 425-427.

CAlle CARABIAS, Q. (1990), La enseñanza oficial de idiomas en Espaha. Por una redefinición de la formación teórica del profesorado, Málaga, Servicio de Publicaciones de la Universidad, (microf.).

CAPITÂN DIAZ, A. (1986), Historia del pensamiento pedagógico en Europa IL Pedagogia contemporánea, Madrid, Dykinson.

ESTEBAN MATEO, L. (1978), Boletín de la Institución Libre de Enseñanza : nómina bibliográfica, Valencia. Junta para Ampliación de Estudios e Investigaciones Cientificas (1925), Un ensayo pedagógico. El Instituto-Escuela de Segunda Enseñanza de Madrid, Madrid.

MOLERO PINTADO, A. (1977), La reforma educativa de la Segunda República española, Madrid, Santillana.

- (1985), La Institución Libre de Enseñanza, un proyecto español de renovación pedagógica, Madrid, Anaya.

PUREN Ch. (1988), Histoire des méthodologies de l'enseignement des langues, Paris, Clé-International.

Roig Morrâs, C. (1989), « Un jardin orné d'acérinées variégates au lycée San Isidro de Madrid », Documents, 4, pp. 29-33.

(1990), « Le français dans les programmes officiels en Espagne : 1934-1938 », Documents, 6, pp. 212-234.

(1994), « Le rôle du français dans l'esprit de quelques Ilustrados espagnols : Feijoo, Jovellanos et Capmany ", Documents, 14, pp. 35-44. 
(1995), « La formation à l'enseignement du FLE en Espagne », Etudes de Linguistique Appliquée, 95, pp. 40-48.

(1998), « L'enseignement du FLE au sein de l'Institución Libre de Enseñanza dans le premier tiers du XXe siècle », Documents, 20, pp. 141-157.

\section{Sources premières}

CASTRO, A. (1921), « La enseñanza de las lenguas modernas », BILE, 733, pp. 119-121.

ESCRICHE Y MIEG, T. (1882), « El método en la enseñanza de las lenguas », BILE, 140, pp. 277-278.

JESPERSEN, O. (1925), « La enseñanza de las lenguas extranjeras », BILE, 788, pp. 321-326 ; 789, pp.

358-370.

JUNTA FACULTATIVA (1882), « La enseñanza comercial », BILE, 124, pp. 82-83.

LANDA, R. (1926), « La enseñanza de las lenguas vivas », BILE, 791, pp. 35-46.

MOORE SMITH, G.C. (1902), « El profesor Jespersen y la enseñanza de las lenguas modernas »

(compte rendu), BILE, 505, pp. 111-112.

PESTANA, A. (1912), « Observaciones sobre la enseñanza del inglés », BILE, 628, pp.193-199; 629, pp.

234-242.

\section{Textes légaux}

(1918), Real Decreto creando el Instituto-Escuela du 10 mai, Gaceîa de Madrid du 11.

(1927), Real Orden du 29 juillet, aprobando el Cuestionario de lenguas vivas, Boletín Oficial del Ministerio de Instrucción Pública du 9 août.

(1931), Decreto del Plan de Estudios du 15 septembre, Gaceta du 16.

, Proyecto de Ley de Bases de la reforma universitaria du 14 mars, Gaceta du 19.

, Decreto del Plan de Estudios du 29 août, Gaceta du 30.

(1934), Real orden du 20 octobre aprobando el Cuestionario de francés, Gaceta du 21.

\section{NOTES}

1. L'Espagne a de plus en plus de relations internationales, et chaque jour le nombre de chaires d'espagnol augmente aux Etats-Unis, en Angleterre, en Italie, en France, etc. Quant à la situation dans la faculté de Lettres, il est honteux de voir qu'elle est incapable de produire une seule page sur une civilisation étrangère, de prononcer un seul mot sur Dante, Shakespeare ou Molière, que ses étudiants reculent effrayés devant un livre en anglais ou allemand, et qu'ils sont rares ceux qui déchiffrent à peu près le français didactique [...]. Le moment ne serait-il pas venu de doter la faculté de Lettres de Madrid d'au moins un enseignement de français digne de ce nom?

2. Je n'ai pas la prétention d'innover, mais uniquement de montrer comment, a mon avis, les méthodes relativement modernes de l'enseignement élémentaire des langues vivantes connues partout, mais ignorées en Espagne, devraient être appliquéesdans nos lycées et écoles normales.

3. Pour acquérir un instrument de culture. Dans ce sens leur valeur est telle qu'on peut affirmer qu'un Espagnol qui ne connaît ni le français, ni l'anglais, ni l'allemand n'est Pas un homme cultivé. C'est ainsi qu'il en était autrefois avec le latin.

4. En donnant à l'enseignement des langues vivantes un caractère exclusivement pratique et en imposant uniquement la méthode directe, elle le place dans une situation d'infériorité face à l'enseignement classique. En effet, il limite ses objectifs à l'acquisition d'un simple instrument, ce 
qui, contrairement à l'enseignement classique, l'empêche d'atteindre des résultats importants dans les domaines de la langue maternelle et de la formation intellectuelle.

5. En dernier ressort, la différence réside dans la valeur accordée à la réflexion pour apprendre quelque chose. Certains disent : on apprend à faire quelque chose en réfléchissant d'abord au sujet de notre action, il faut penser à la façon de faire, connaître les concepts, et les lois générales relatives à l'activité en question, c'est-à-dire, sa théorie. D'autre part on dit : on apprend à faire quelque chose en observant ceux qui le font et en le faisant ; ce n'est pas la connaissance de la théorie, mais la pratique ; ce n'est pas la réflexion au sujet de l'action, mais l'action en elle-même qui nous apprend à faire [...]. La répétition et l'habitude, et non la connaissance ni la réflexion nous apprend à faire, il en est de même pour parler une langue que pour jouer du Piano ou nager. 6. Quand les autres nommaient quelque chose et quand d'après leur parole ils se déplaçaient vers quelque chose, je les observais et retenais que lorsqu'ils prononçaient ce mot, ils étaient en train de nommer l'objet qu'ils voulaient montrer Ainsi, replaçant ces mots dans leur contexte à plusieurs reprises, et à force de les écouter, $\mathrm{j}$ 'arrivais petit à petit à comprendre leur sens et, une fois ma bouche habituée à la prononciation de ces signes, $\mathrm{j}$ 'exprimais avec eux ce que je voulais.

7. L'essentiel est que l'élève ait assimilé la structure de la langue, qu'il pense dans la langueétrangère, et qu'il soit capable spontanément comme par un instinct grammatical, de construire des phrases dans l'ordre correct, et de se servir des notes variables avec les modifications qui conviennent selon lecas.

8. Moins on emploiera de temps à l'acquisition de l'instrument, et plus à la maîtrise de la traduction, mieux ce sera. La méthode directe qui demande un travail purement mécanique, sans aucune valeur formative, ne doit être appliquée que le temps strictement indispensable.

9. Daniel Jones (1881-1967), alors Secrétaire adjoint de l'API.

\section{AUTHOR}

\section{CARMEN ROIG MORRÁS}

Universidad de Cantabria 